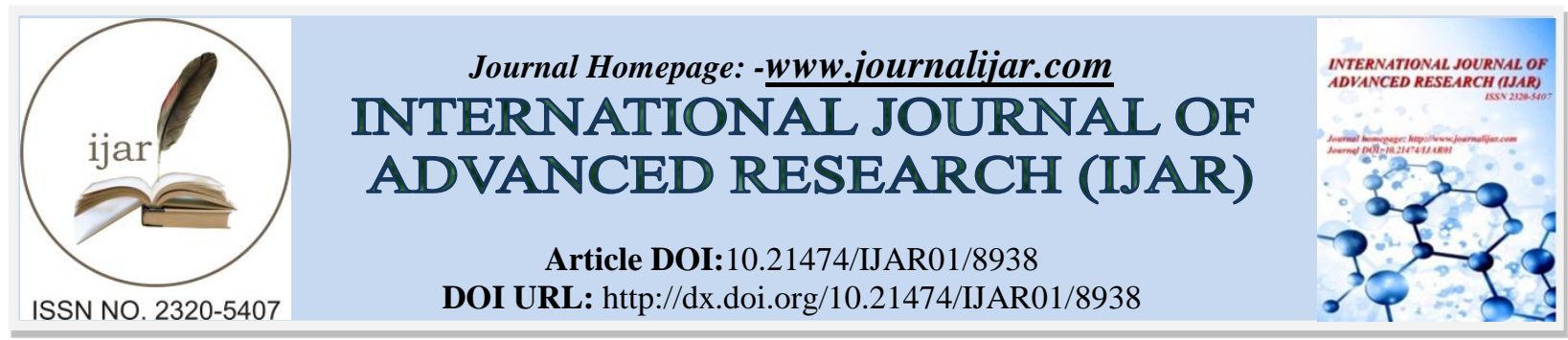

RESEARCH ARTICLE

\title{
POLYCYSTIC OVARIAN SYNDROME IN ADOLESCENT AGE GROUP AT TERTIARY CARE CENTRE.
}

Manra singh ${ }^{1}$ And Usha rawat ${ }^{2}$.

1. PG resident third year, Obs and Gynae Department, Susheela Tiwari Govt. Hospital, Haldwani, Uttarakhand.

2. Professor, Obs and Gynae Department, Susheela Tiwari Govt. Hospital, Haldwani, Uttarakhand.

\section{Manuscript Info}

Manuscript History

Received: 22 February 2019

Final Accepted: 24 March 2019

Published: April 2019

\section{Abstract}

Polycystic ovarian syndrome is one of the gynaecological problems during adolescence. It is one of the most common endocrine disorder affecting $5-10 \%$ women worldwide ${ }^{(4)}$. This study was conducted to study the clinical and biochemical features of PCOS because it is important to screen and diagnose adolescent PCOS in order to prevent the development of future infertility, type II diabetes mellitus, cardiovascular disease, metabolic syndrome and endometrial cancer in PCOS patients.

Material and methods: Cross sectional study conducted on 50 patients attending the OPD and IPD at Dr. Susheela Tiwari Govt. Hospital for menstrual irregularities and other gynaecological problems.

Conclusion: Out of the PCOS patients studied at Susheela Tiwari Hospital , Haldwani,25\% were overweight and $8 \%$ were obese, residing in urban areas, Waist hip ratios of $62 \%$ patients in the study group were $>0.80$. $62 \%$ patients had menstrual irregularities and $44 \%$ patients had clinical features of Hyperandrogenism. However, biochemical Hyperandrogenism was seen only in $10 \%$ of patients. $20 \%$ and $12 \%$ patients had a family history of Diabetes Mellitus and Hypertension respectively. Hypothyroidism also accompanied a small percentage of patients with PCOS, the percentage being $20 \%$. LH/FSH ratio was raised in $14 \%$ patients and ultrasound features of PCOS were found in a large group of patients, the percentage being $60 \%$.

Copy Right, IJAR, 2019,. All rights reserved.

\section{Introduction:-}

Adolescence is a transitional stage of physical and psychological development that generally occurs during the period from puberty to adulthood that lies in the age group of 10-19 years (as per WHO guideline) (1) (2) (3). Adolescent girls face many gynaecological problems like

1. Precocious puberty

2. Delayed puberty

3. Oligomenorrhoea

4. Polymenorrhoea

5. Pubertal menorrhagia

6. Dysmenorrhoea

Corresponding Author:-Manra singh. 
Polycystic ovarian syndrome is one of the gynaecological problems during adolescence. It is one of the most common endocrine disorder affecting 5-10\% women worldwide ${ }^{(4)}$.

It is a challenging public health problem affecting 1 in 5 women of reproductive age group ${ }^{(5)}$ and is a frustrating experience for women.

Polycystic Ovarian Syndrome was originally described in 1935 by Stein and Leventhal as a syndrome manifested by Amenorrhea, Hirsutism and Obesity associated with enlarged polycystic ovaries ${ }^{(4)}$.

It affects $20-30 \%$ of reproductive age group women and 30\% of regularly menstruating girls. The common symptoms include irregular menses, cutaneous disorders (acne, hirsutism) and obesity ${ }^{(6)(7)}$. Adolescent girls require long term committed treatment so that they don't land up in infertility in future

This heterogeneous disorder is characterized by excessive androgen production by ovaries and is a multifactorial and polygenic condition.

Diagnosis is based on the presence of two of the following three criteria

ESHRE/ASRM Statement (Rotterdam 2003) ${ }^{(8)}$

1. Oligo-ovulation/ an ovulation

2. Hyperandrogenism (clinical and biochemical)

3. Polycystic ovaries in ultrasonography

(ESHRE: European Society For Human Reproduction And Embryology)

(ASRM: American Society For Reproductive Medicine)

Typically the ovaries are enlarged ( $>10 \mathrm{cc}$ in volume) and there is presence of multiple ( $>12$ folicular cysts measuring 2-9 $\mathrm{mm}$ in diameter) crowded around the cortex.

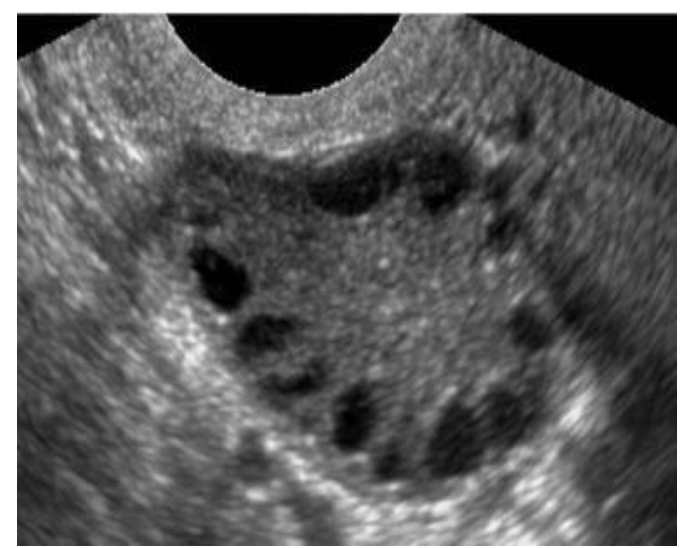

Figure 1:-Transvaginal Ultrasound Showing Peripheral Distribution Of Follicles

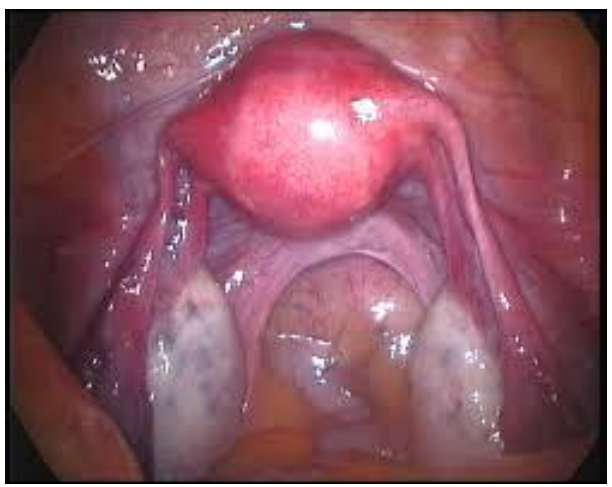

Firgure 3:-Laproscopic appearence of polycystic ovary 
In this Syndrome, due to insulin resistance, there is Hyperinsulinemia which participates in the ovarian hormone dysfunction. Insulin alters the ovarian steroidogenesis independent of gonadotropin secretion ${ }^{(8)}$.

Insulin increases the ovarian androgen synthesis by increasing LH pulse frequency at Pituitary gland and due to insulin resistance there is risk of gestational diabetes, Type 2 Diabetes Mellitus, obstructive sleep apnea, cardiovascular disease, endometrial cancer and poor quality of life. Insulin inhibits the hepatic synthesis of sex hormone binding globulin the main circulating protein that binds to testosterone, thus increasing the production of unbound or bioavailable testosterone ${ }^{(8)}$.

Acanthosis nigricans is a reliable marker of insulin resistance in hirsute women. This thickened, velvety, pigmented lesion is most often found in the vulva, may be present in the axilla, over the nape of neck, below the breast and on the inner thigh.

1. HAIR- AN syndrome consists of

2. Hyperandrogenism (HA)

3. Insulin resistance (IR) and

4. Acanthosis nigricans (AN).

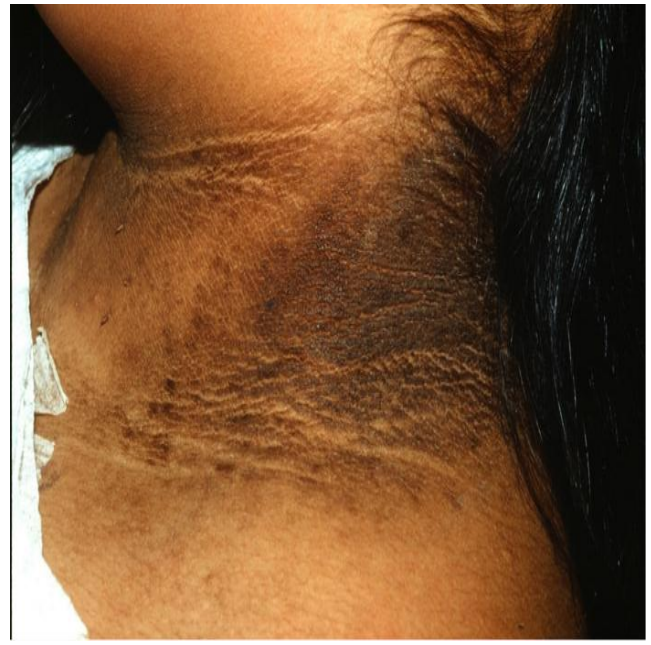

Figure 4:-acanthosis nigricans

Being a tertiary care centre a lot of adolescent girls attend the OPD with menstrual irregularities and other features associated with PCOS. This study was conducted to study the clinical and biochemical features of PCOS because it is important to screen and diagnose adolescent PCOS in order to prevent the development of future infertility, type II diabetes mellitus, cardiovascular disease, metabolic syndrome and endometrial cancer in PCOS patients.

Although adolescent's concerns are often cosmetic, if left untreated these girls are at risk for diabetes, metabolic syndrome, and infertility as they mature. Efforts have been made to diagnose and treat PCOS to minimize the development of symptoms and prevent the onset of cardiovascular and metabolic disturbances.

\section{Material And Methods:-}

StudyDesign

Cross sectional

\section{Sample Size}

Study was conducted on 50 adolescent girls attending Obstetrics and Gynaecology OPD and IPD at Dr. Susheela Tiwari Government Hospital for menstrual irregularities and other gynaecological problems .

\section{Methodology:-}

Written and informed consent was obtained from the all the adolescent patients and their parents/guardians agreeing to participate in the study and confidentiality was maintained. All patients were subjected to detailed clinical history, menstrual and family history. Height and Weight were measured and BMI was calculated. Detailed history was 
followed by Ultrasonography for uterus and adnexa. Hormone profile was done on all adolescent girls (preferably on day 2 or day 3 of menstrual cycle) which included:

Leutinising hormone (LH) , Follicle stimulating hormone(FSH) ,LH :FSH ,Dehydroepiandrostenedione Sulphate(D.H.E.A.S.) ,Androstenedione, Total and free testosterone, Dihydrotestosterone(DHT), Serum insulin (fasting )

If any of the 2 out of 3 criteria are fulfilled, patient was considered as a case of PCOS

ESHRE/ASRM Statement (Rotterdam 2003)

1. Oligo-ovulation/ anovulation

2. Hyperandrogenism (clinical and biochemical)

3. Polycystic ovaries

(ESHRE: European Society For Human Reproduction and Embryology)

(ASRM: American Society For reproductive Medicine)

If none of the criteria for PCOS were fulfilled, patient was investigated to rule out other causes of menstrual irregularities.All patients were subjected to routine blood investigations.

\section{Result:-}

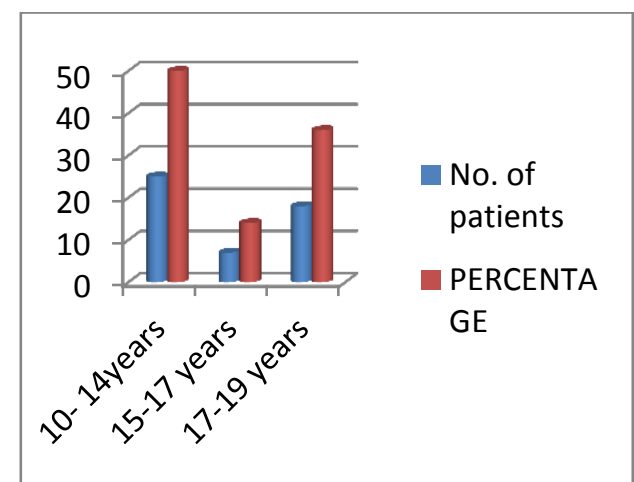

Chart 1:-Relation Between Age And Pcos

$50 \%$ patients lie between the age group of 10-14 years, $14 \%$ lie between the age group of $15-16$ years and 36\% lie between the age group of 17-19 years.

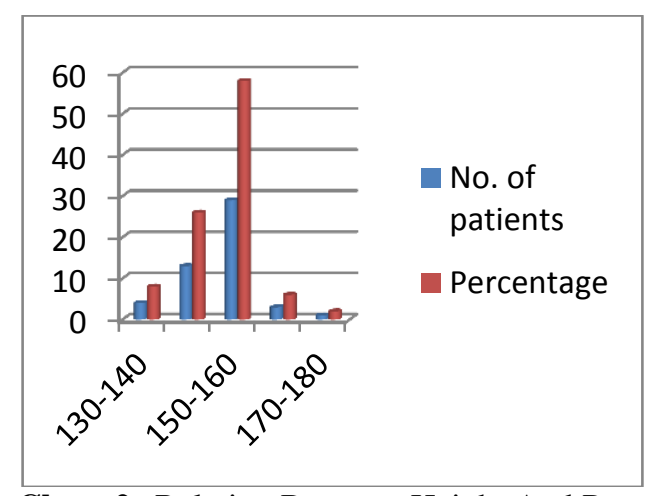

Chart 2:-Relation Between Height And Pcos

Patients having height between $130-140 \mathrm{~cm}$ were (8\%), between $140-150 \mathrm{~cm}(26 \%)$, between $150-160 \mathrm{~cm}(58 \%)$, between 160-170(6\%) and between 170-180 (2\%). 


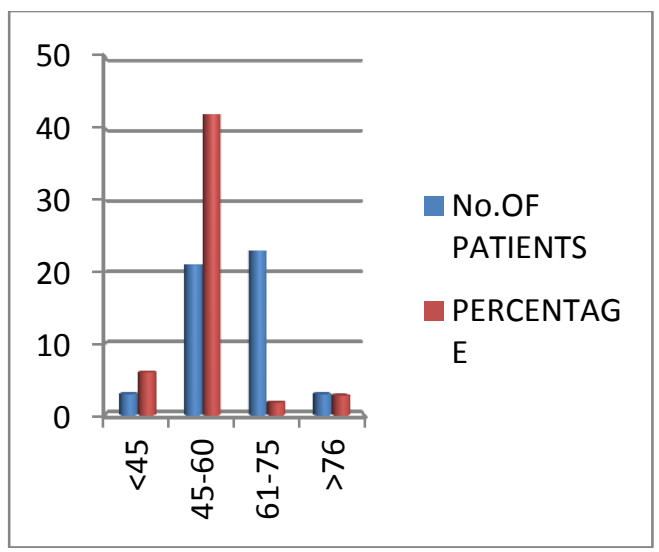

Chart 3:-Relation Between Weight And Pcos

$6 \%$ patients have weight less than $45 \mathrm{Kg} .42 \%$ patients have weight between $46-60 \mathrm{Kg} .46 \%$ patients have weight between $61-74 \mathrm{Kg} .6 \%$ patients have weight more than $75 \mathrm{Kg}$.

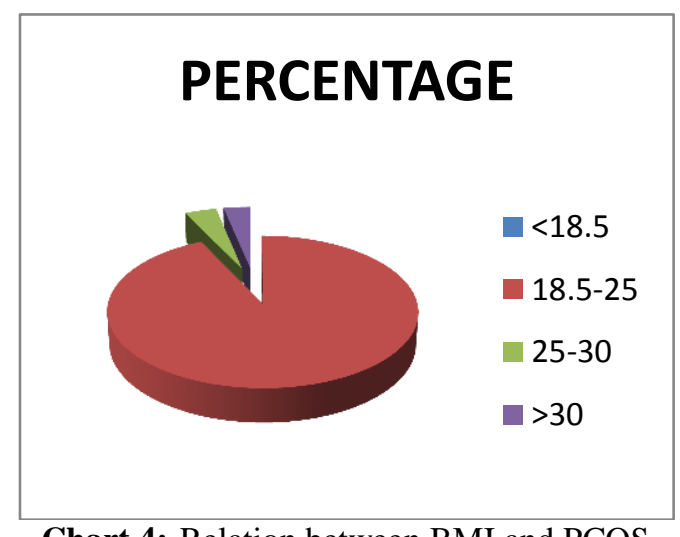

Chart 4:-Relation between BMI and PCOS

None of the patients have BMI less than 18.5, 34\% patients have BMI between 18.5-25, 50\% patients have BMI between $25-30$ and $16 \%$ patients have BMI more than 30

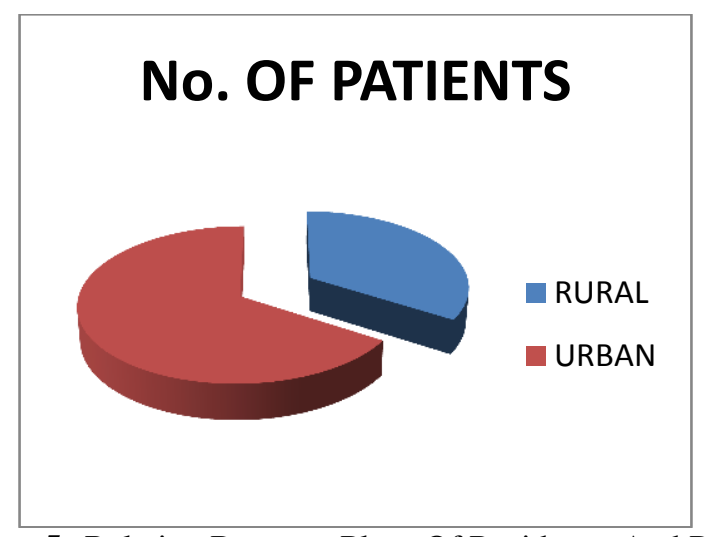

Chart 5:-Relation Between Place Of Residence And Pcos.

The number of PCOS patients residing in urban areas was $66 \%$ whereas those residing in rural areas were $34 \%$ 


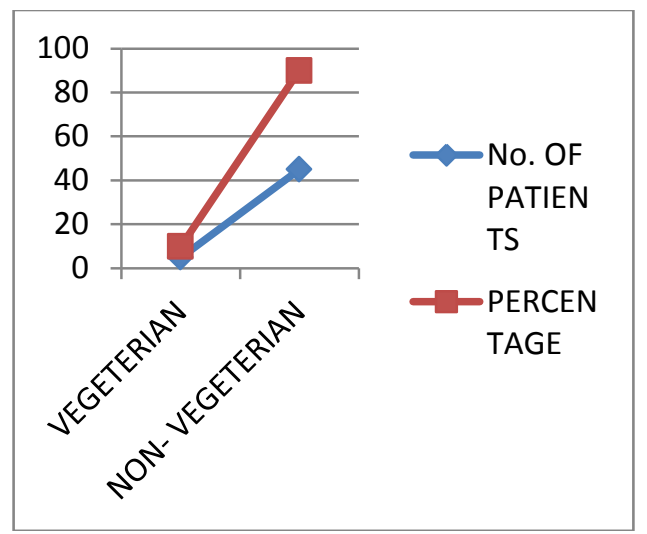

Chart 6:-Relation Between Dietary Pattern And Pcos

Number of patients eating non-vegetarian food were $90 \%$ and those eating vegetarian food were $10 \%$.

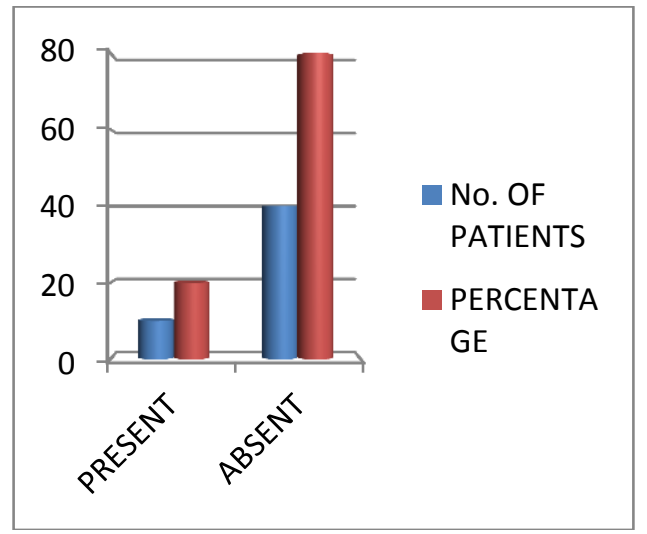

Chart 7:-Relation Between Family History Of Diabetes Mellitus

$20 \%$ if PCOS patients in the study had a family history of Diabetes Mellitus and $80 \%$ patients did not have a family history of Diabetes Mellitus

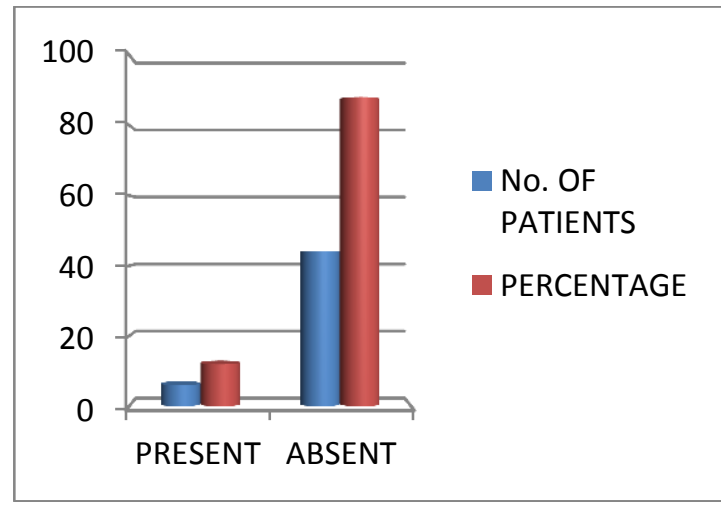

Chart 8:-Relation Between Family History Of Hypertension And Pcos.

$12 \%$ of PCOS patients in this study had a family history of Hypertension and $88 \%$ patients did not have a family history of PCOS. 


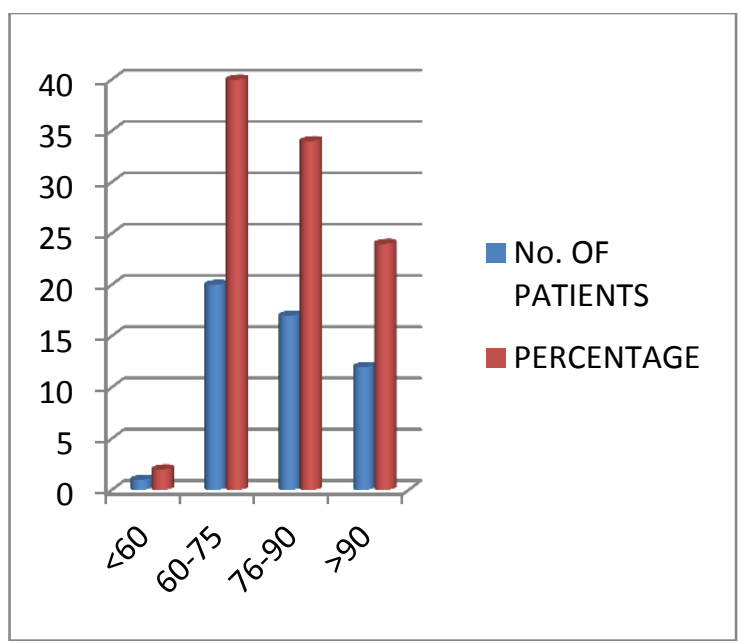

Chart 9:-Relation Between Waist Circumference And Pcos

$2 \%$ patients had waist circumference less than $60 \mathrm{~cm}, 40 \%$ patients had waist circumference between $61-75 \mathrm{~cm}$, $34 \%$ patients had waist circumference between $76-90 \mathrm{~cm}$ and $24 \%$ patients had waist circumference more than $90 \mathrm{~cm}$.

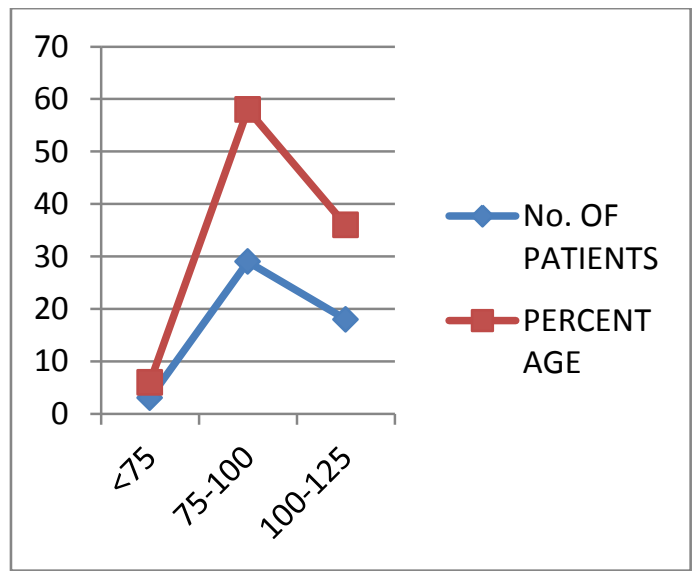

Chart 10:-Relation Between Hip Circumference And Pcos

$6 \%$ patients had hip circumference less than $75 \mathrm{~cm}, 58 \%$ patients had hip circumference between $76-100 \mathrm{~cm}$. and $36 \%$ patients ha hip circumference between $100-125 \mathrm{~cm}$

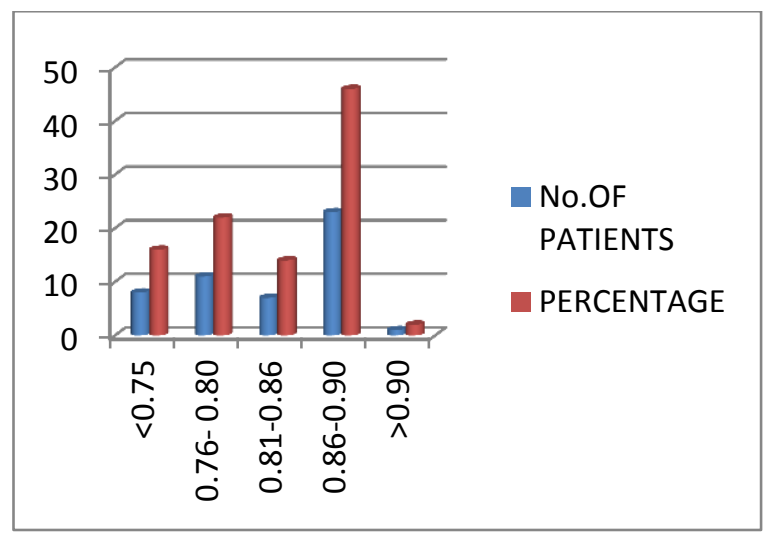

Chart 11:-Relation Between Waist Hip Ratio And Pcos 
In this study $16 \%$ patients had waist hip ratio less than $0.75,22 \%$ had waist hip ratio between $0.75-0.80,14 \%$ had waist hip ratio between $0.81-0.85,46 \%$ had waist hip ratios between $0.86-0.90$ and $2 \%$ had waist hip ratios more than 0.90 .

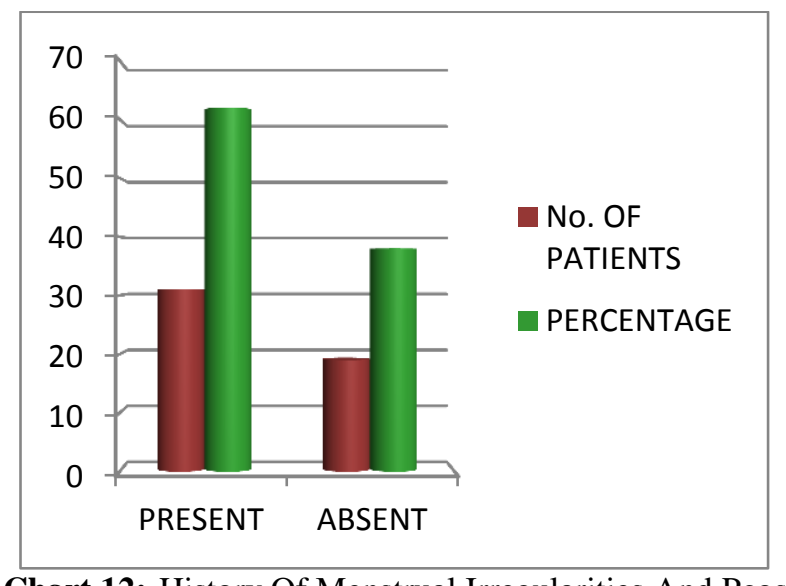

Chart 12:-History Of Menstrual Irregularities And Pcos

$62 \%$ patients of PCOS in this study had history of menstrual irregularities and $38 \%$ did not have any history of menstrual irregularities.

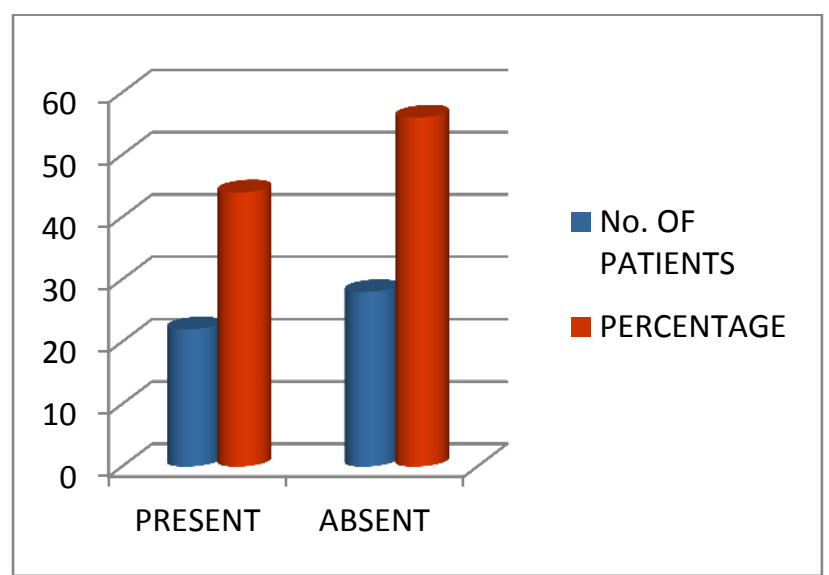

Chart 13:-Relation Between Clinical Features Of Hyperandrogenism And Pcos.

$44 \%$ of PCOS patients in this study showed clinical features of Hyperandrogenism. The other $56 \%$ had no clinical features of Hyperandrogenism

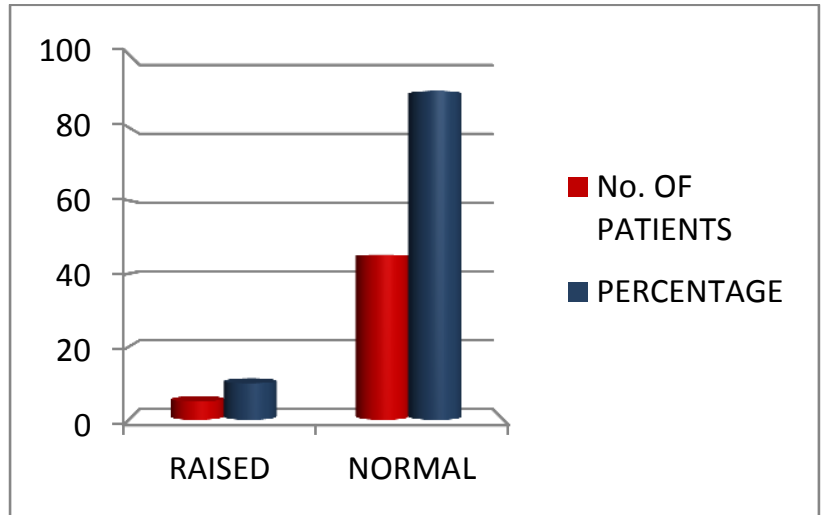

Chart 14: Relation Between Raised Ogtt And Pcos. 
$10 \%$ PCOS patients in this study had raised OGTT and 90\% patients had normal OGTT values

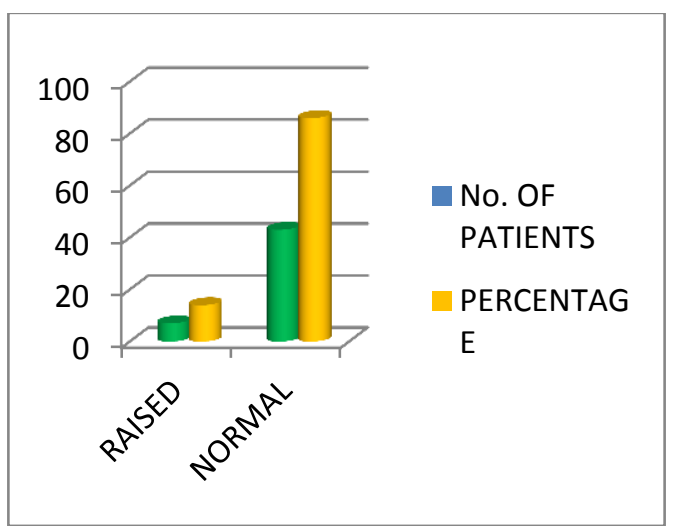

Chart 15:-Relation Between Lh/Fsh Ratio And Pcos.

In this study $14 \%$ patients had raised LH/FSH ratio whereas the other $86 \%$ had normal LH/FSH ratio

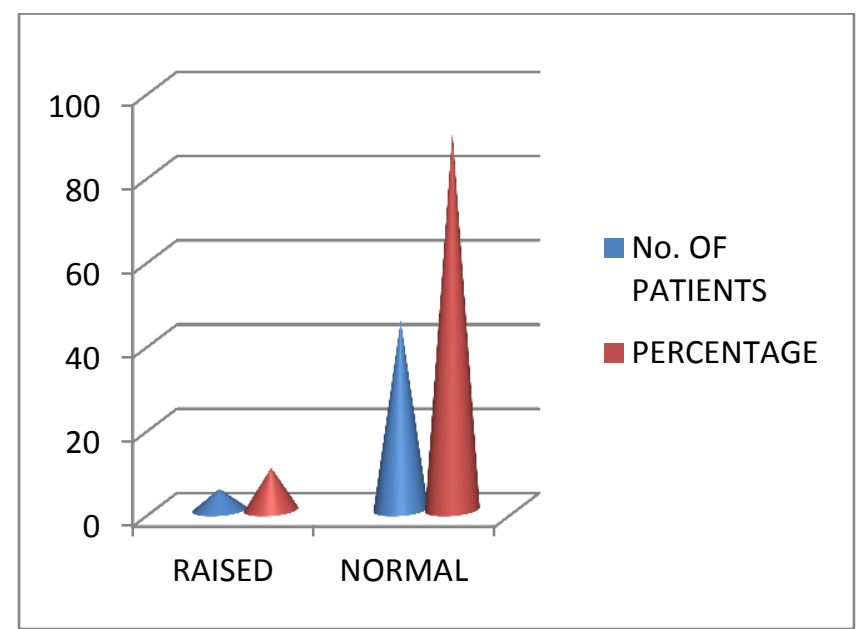

Chart 16:-Relation Between Serum Testosterone And Pcos

In this study $10 \%$ of PCOS patients had raised Serum Testosterone levels whereas the other $90 \%$ patients had normal serum testosterone levels.

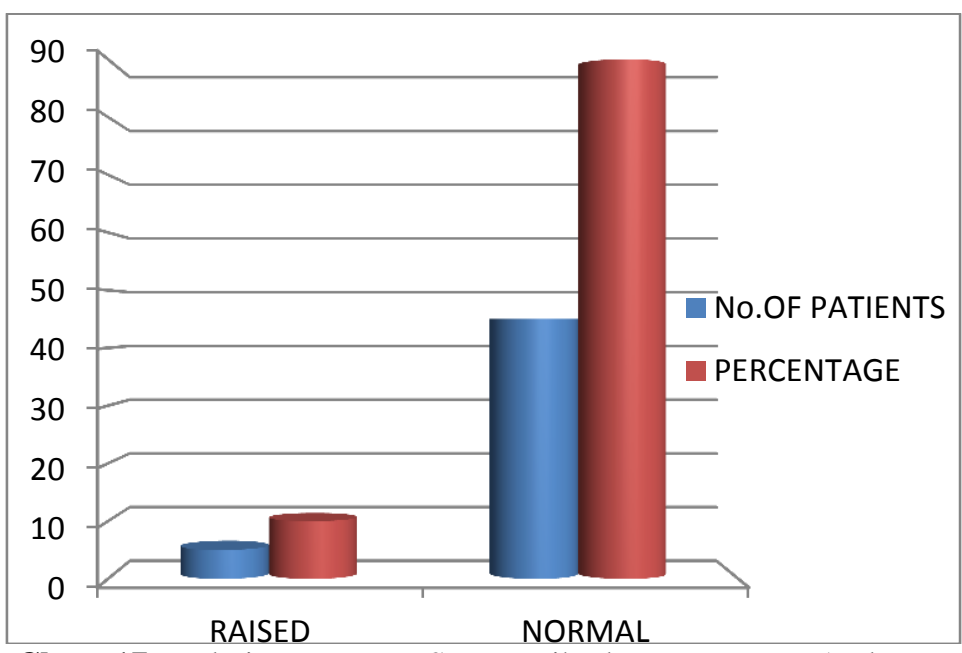

Chart 17:-Relation Between Serum Dihydrotestosterone And Pcos 
In this study $10 \%$ of PCOS patients had raised Serum Dihydrotestosterone levels and the other $90 \%$ had normal Serum Dihydrotestosterone levels.

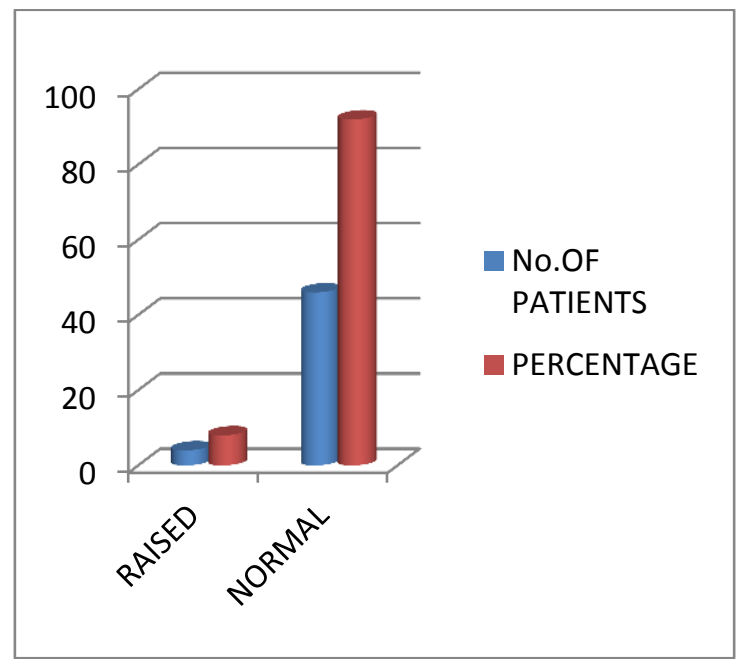

Chart 18:-Relation Between Serum Dheas And Pcos

In this study 8\% patients had raised Serum DHEAS levels and 92\% had Serum DHEAS values normal.

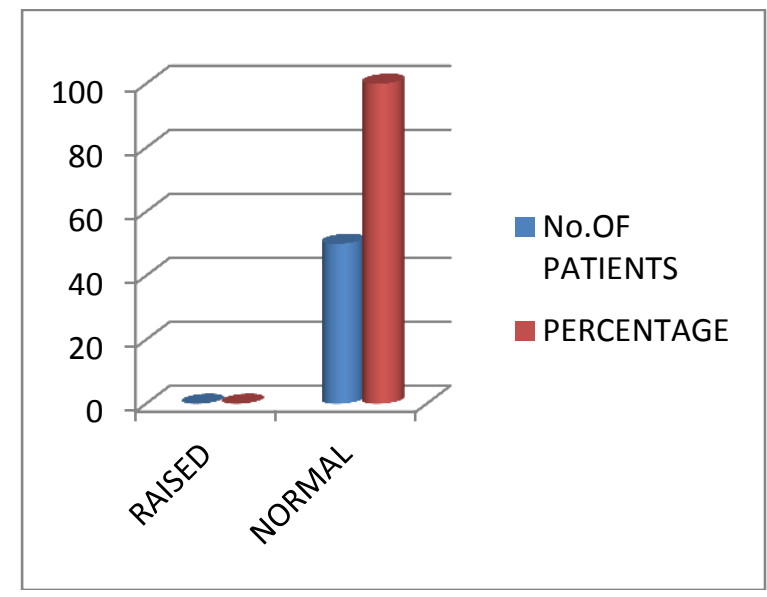

Chart 19:-Relation Between Serum Prolactin Levels And Pcos

In this study all the patients had normal serum prolactin levels

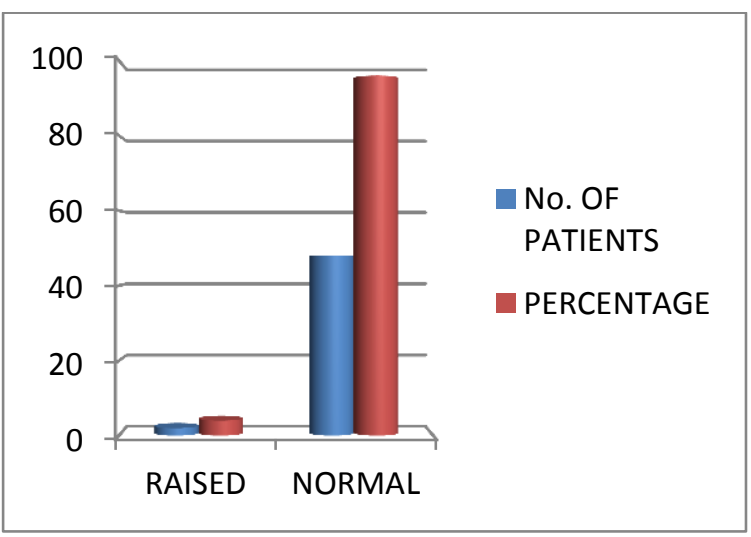

Chart 20:-Relation Between Serum Fasting Insulin And Pcos 
In this study 4\% patients had raised Serum Prolactin levels whereas 96\% had normal serum prolactin levels.

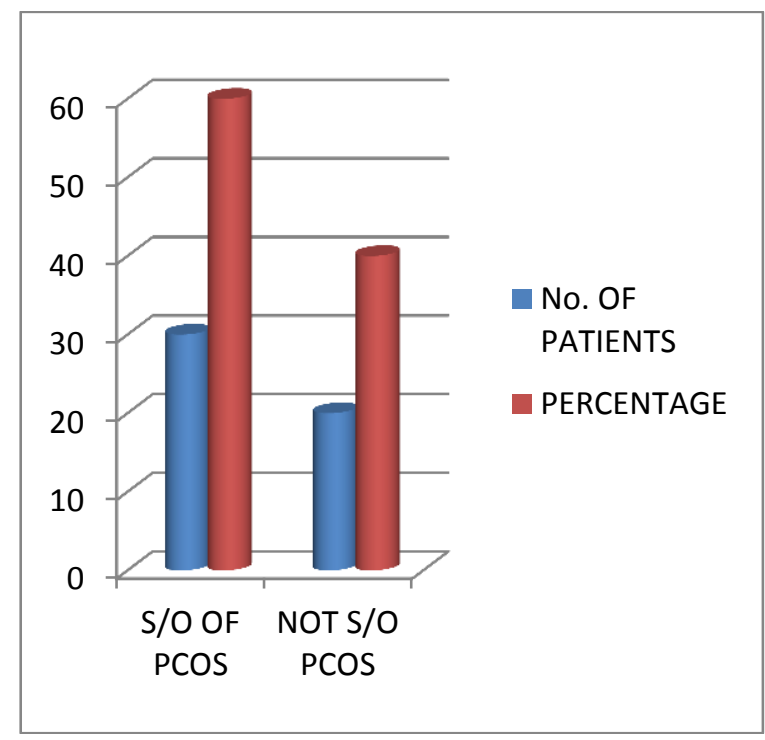

Chart 21:-Ultrasound Suggestive Of Pcos

In this study $60 \%$ patients had ultrasound features suggestive of PCOS. In $40 \%$ patients there were no ultrasound features suggestive of PCOS.

\section{Summary And Conclusion:-}

1. Out of the PCOS patients studied at Susheela Tiwari Hospital, Haldwani,25\% were overweight and $8 \%$ were obese.

2. Most of the patients who had PCOS were residing in urban areas. This is also supported by other studies which explain higher incidence of PCOS in urban population as a result of lifestyle changes.

3. $90 \%$ of the PCOS patients in the study were non vegetarian.

4. In this study $20 \%$ and $12 \%$ patients had a family history of Diabetes Mellitus and Hypertension respectively.

5. Waist hip ratios of $62 \%$ patients in the study group were $>0.80$ which explains the correlation between central obesity and PCOS. Some studies have also shown that presence of central obesity worsens the clinical and biochemical profile of PCOS patients.

6. In this study $62 \%$ patients had menstrual irregularities and $44 \%$ patients had clinical features of Hyperandrogenism. However, biochemical Hyperandrogenism was seen only in $10 \%$ of patient explaining that biochemical and clinical hyperandrogenism in PCOS are distinct entities and no androgen correlates well with clinical hyperandrogenism, acne, or hirsutism.

7. OGTT being a screening tool for future predisposition for Diabetes Mellitus was deranged in $10 \%$ patients only.

8. Hypothyroidism also accompanied a small percentage of patients with PCOS, the percentage being $20 \%$.

9. In this study, LH/FSH ratio was raised in $14 \%$ patients. The results show that LH/FSH ratio is not a characteristic attribute of all PCOS women which is also proved by some other studies.

10. Serum prolactin levels were within normal limits for all the patients included in this study.

11. In this study, ultrasound features of PCOS were found in a large group of patients, the percentage being $60 \%$. Ultrasound is an easy and cost effective tool for diagnosis of PCOS. Ultrasound finding of PCOS thus can be followed up with hormonal profiles and both can be correlated clinically.

12. Thus, we conclude that best efforts have been made in this study to identify the clinical and biochemical features of PCOS in order to screen and diagnose adolescent PCOS to prevent the development of future infertility, type II diabetes mellitus, cardiovascular disease, and even endometrial cancer.

13. Efforts have been made to diagnose and treat PCOS to minimize the development of symptoms and prevent the onset of cardiovascular and metabolic disturbances. 


\section{References:-}

1. Macmillan dictionary for students Macmillan, pan ltd. (1981), page 14, 456. Retrieved 2010-7-15.

2. "Adolescence". Merriam-webster. Retrieved may 9, 2012.

3. Shaws textbook of gynaecology

4. Azziz r, woods ks, reyna $\mathrm{r}$, key $\mathrm{tj}$, knochenhauer es, yildiz bo. the prevalence and features of the polycystic ovary syndrome in an unselected population. $j$ clin endocrinol metab. 2004;89:2745-2749.[pubmed]

5. http://www.nichd.nih.gov. Archived from the original on 3 march 2015. Retrieved 13 march 2015.

6. Dutta gynae $7 / \mathrm{e}$

7. Berek and novak's gynaecology $15 / \mathrm{e}$

8. Kriplani \& Agarwal, 2004 metformin \& pcos improvement in menstrual cyclicity. 